\title{
Resource Allocation and User Association for Load Balancing in NOMA-Based Cellular Heterogeneous Networks
}

\author{
Sima Sobhi-Givi ${ }^{1}$, Mahrokh G. Shayesteh ${ }^{1,2}$, Hashem Kalbkhani ${ }^{3}$, and Nandana Rajatheva ${ }^{4}$ \\ ${ }^{1}$ Department of Electrical and Computer Engineering, Urmia University, Urmia, Iran \\ ${ }^{2}$ Wireless Research Lab, ACRI, Elec. Eng. Dep., Sharif University of Technology, Tehran, Iran \\ ${ }^{3}$ Faculty of Electrical Engineering, Urmia University of Technology, Urmia, Iran \\ ${ }^{4}$ Centre for Wireless Communications (CWC), University of Oulu, Finland \\ Emails: s.sobhi@urmia.ac.ir,m.shayesteh@urmia.ac.ir,h.kalbkhani@uut.ac.ir,nandana.rajatheva@oulu.fi
}

\begin{abstract}
Small cells, specially femtocells, are deployed in the coverage of macrocells and form underlay heterogeneous networks (HetNets) to increase the capacity of cellular networks. Load balancing is necessary to increase the resource utilization in HetNets. Non-orthogonal multiple access (NOMA) technique has been proposed to increase the spectral efficiency in the fifthgeneration (5G) of cellular networks. The aim of this paper is to present a user association and resource block (RB) allocation scheme in NOMA-based cellular HetNet for maximization of Jain's fairness index and spectral efficiency. The maximization problem is decomposed into two sub-problems. At first, the number of RBs that each small cell base station (SBS) can allocate for connection requests, is obtained. Then, the NOMA groups and RB allocation are optimized to increase the spectral efficiency. The results indicate the efficiency of the proposed scheme in enhancing the fairness index and spectral efficiency in HetNets compared to the conventional scheme.
\end{abstract}

Keywords-Heterogeneous network (HetNet), load balancing, non-orthogonal multiple access (NOMA), resource block (RB), spectral efficiency, user association.

\section{INTRODUCTION}

The increasing demand for wireless services and the need for high data rates result in heterogeneity of base stations (BSs) with different transmit powers, coverage ranges, and costs of deployment. Deploying small cells such as picocells and femtocells in heterogeneous networks (HetNets) improves spectrum efficiency. Sharing resources among multi-tier cells causes cross-tier interference. Thus, efficient resource allocation and interference management algorithms are required to overcome this issue, which is one of the fundamental research challenges in HetNets [1]. In the fifthgeneration $(5 \mathrm{G})$ of wireless networks, non- orthogonal multiple access (NOMA) has been considered as one of the most important methods in designing radio access techniques. NOMA techniques in 5G include power domain NOMA, sparse code multiple access (SCMA), low density spreading (LDS), and lattice partition multiple access (LPMA). These techniques serve more than one user in each orthogonal resource block (RB), while conventional orthogonal multiple access (OMA) techniques serve only one user in each orthogonal RB [2]. Sharing one RB by several users in NOMA enhances spectrum efficiency.
User association is a challenging task in NOMA-based dense HetNets. Efficient user association algorithms should balance the load among the BSs and avoid congested cells to enhance the spectrum and energy efficiencies of the network [3]. In HetNets, in addition to load balancing among small cell base station (SBSs), it is preferred that the traffic of macrocell base station (MBS) is offloaded to SBSs [4]. Interference from the nearby macrocells to the users associated with small cells, is one of the most important challenges in load balancing [5]. Therefore, the improvement achieved by offloading traffic to small cells, might be influenced by strong interference. Hence, the trade-off between the load balancing and interference depends on the value of the selected bias, which has to be carefully optimized in order to maximize the network utility [6].

The problem of optimal user association in a mmWave multiple-input multiple-output (MIMO) cellular network was studied in [7]. First, the activation matrix was introduced and it was shown that the instantaneous rate of users is a function of the activation matrix. Then, a user association scheme was investigated which considers the effect of user association on the interference. In [8], load balancing in HetNet was studied for improving the capacity of the network. In [9], the minimization of the latency of requested services in femtocell networks was formulated for user association and it was solved by several approximated algorithms. In [10], the maximization of minimum data rate was obtained by joint user association and channel allocation between macrocells and small cells. Joint user association and power control was investigated for HetNets in the downlink in [11-13] and uplink in $[14,15]$. The methods introduced in [11-14] obtained the solution of user association and power allocation by using the iterative algorithms. The sum throughput maximization problem was formulated as a non-cooperative game in [15] in which users and BSs act as players. In [16], user association, spectrum allocation, and interference coordination were studied for enhancing the capacity of HetNets. In the mentioned works, OMA-based techniques were considered. NOMA-based user association for load balancing in HetNets was considered in [4], where SBS tier was used NOMA and carrier sensing for transmission. The carrier sensing was modelled using a repulsive point process. For load balancing, the users of the MBS tier were offloaded to SBSs.

In this paper, we consider user association and RB allocation problem in NOMA-based HetNets for maximization of spectral efficiency and Jain's fairness index, which is a 
measure of load balancing. SBSs use both OMA and NOMA techniques to serve the users. To this end, the maximization problem is decomposed into two sub-problems, where their aim is to maximize the fairness index and spectral efficiency. We propose a mobile-assisted network-controlled (MANC) solution to solve the sub-problems. To this end, each user specifies its neighbor SBSs based on the reference signal received power (RSRP) and then, sends a connection request to the desired SBS in the neighbor list. Considering the received connection requests and the number of RBs that SBS can allocate to users, SBS decides whether to form the NOMA groups or serve users in OMA mode. The results show that the proposed scheme enhances the fairness index and spectral efficiency in NOMA-based HetNets and outperforms the conventional RSRP-based scheme.

The rest of this paper is organized as follows. Section II describes the system model used in this study. Section III presents the proposed user association. Simulation results are provided in Section IV and Section V concludes the paper.

\section{SYSTEM MODEL}

\section{A. HetNet Model}

The HetNet is this paper includes one macrocell and several small cells. SBSs are located at the center of the cell and can serve the users in both OMA and NOMA modes with omnidirectional antennas. SBSs are distributed according to the spatial Poisson point process (SPPP) distribution with the density $\lambda_{F}$ in the coverage area of macrocell. The set of $N_{F}$ SBSs is denoted by $\mathbf{F}=\left\{f_{1}, f_{2}, \ldots, f_{N_{F}}\right\}$. Also, users are distributed according to the uniform distribution with the density $\lambda_{u}$. The set of $N_{u}$ users is represented with $\mathbf{U}=\left\{u_{1}, u_{2}, \ldots, u_{N_{u}}\right\}$. It is assumed that each SBS has $N_{b}$ RBs which are randomly chosen from the available RBs.

SBSs operate in hybrid mode, where some RBs are allocated to the dedicated users and the remaining ones can be assigned for ongoing requests from other users. According to Fig. 1, some of the requesting users are grouped in two-user NOMA groups, some users are served via OMA mode, and connection request of some users is rejected by the SBS in admission control process and these users should send connection request to other SBSs.

\section{B. Channel Model}

In the wireless channel, the transmit power is affected by three impairments including path loss $(L)$, shadowing $(\psi)$, and multi-path fading $(h)$. Shadowing follows zero-mean lognormal distribution with standard deviation $\sigma$. Also, channel power gain has unit-mean exponential distribution. Considering the overall effect of impairments, the RSRP of the user $u_{v}$ served by the SBS $f_{k}$ in the RB $b_{j}$ via OMA mode is obtained as:

$$
P_{v, j, k}^{O}=P_{t} h_{v, j, k} \psi_{v, j, k} L_{v, j, k}^{-1}
$$

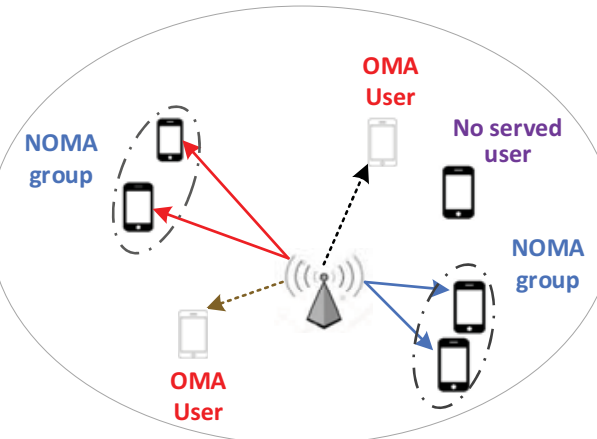

Fig. 1. Demonstration of user association and forming NOMA groups.

where $P_{v, j, k}^{O}$ and $P_{t}$ denote the RSRP in OMA mode and SBS transmit power, respectively. The signal to interference and noise ratio (SINR) of OMA user $\left(\gamma_{v, k, j}^{O}\right)$ is calculated as:

$$
\gamma_{v, j, k}^{O}=\frac{P_{t} h_{v, j, k} \psi_{v, j, k} L_{v, j, k}^{-1}}{P_{v, j, k}^{i}+N_{0}}
$$

where $N_{0}$ represents the white noise power and $P_{v, j, k}^{i}$ is the cochannel interference from other transmissions calculated as:

$$
P_{v, j, k}^{i}=\sum_{j=1}^{N_{F}} x_{j, k} P_{t} h_{v, j, k} \psi_{v, j, k} L_{v, j, k}^{-1}
$$

where $x_{j, k}$ is a binary variable that represents the status of the $\mathrm{RB} b_{j}$ in the SBS $f_{k}$. If SBS $f_{k}$ allocates RB $b_{j}$ to an OMA user or NOMA users, we have $x_{j, k}=1$, otherwise, $x_{j, k}=0$.

On the other side, if the user $u_{s}$ is considered as the strong user of NOMA group in the RB $b_{c}$ of the SBS $f_{k}$, its RSRP is calculated as:

$$
P_{s, c, k}^{N, s}=\beta_{s} P_{t} h_{s, c, k} \psi_{s, c, k} L_{s, c, k}^{-1}
$$

where $0<\beta_{s}<0.5$ is the transmit power gain for the strong user. Similarly, the RSRP of the user $u_{w}$ as the weak user is obtained as:

$$
P_{w, c, k}^{N, w}=\beta_{w} P_{t} h_{w, c, k} \psi_{w, c, k} L_{w, c, k}^{-1}
$$

where $\beta_{w}=1-\beta_{s}>\beta_{s}$ is the transmit power gain for the weak user.

For NOMA users, considering the successive interference cancellation (SIC) at the receivers, at first, the strong user decodes the signal of weak user and then, decodes its own signal. Thus, the SINR of weak user at the strong user $\left(\gamma_{w, c, k}^{N, w \rightarrow s}\right)$ will be equal to:

$$
\gamma_{w, c, k}^{N, w \rightarrow s}=\frac{\beta_{w} P_{t} h_{s, c, k} \psi_{s, c, k} L_{s, c, k}^{-1}}{P_{s, c, k}^{i}+\beta_{s} P_{t} h_{s, c, k} \psi_{s, c, k} L_{s, c, k}^{-1}+N_{0}}
$$

Also, the SINR of strong user $\left(\gamma_{s, c, k}^{N, s}\right)$ can be represented as: 


$$
\gamma_{s, c, k}^{N, s}=\frac{\beta_{s} P_{t} h_{s, c, k} \psi_{s, c, k} L_{s, c, k}^{-1}}{P_{s, c, k}^{i}+N_{0}}
$$

Similarly, weak user decodes its own signal by considering the signal of strong user as noise. Therefore, its $\operatorname{SINR}\left(\gamma_{w, c, k}^{N, w}\right)$ can be expressed as:

$$
\gamma_{w, c, k}^{N, w}=\frac{\beta_{w} P_{t} h_{w, c, k} \psi_{w, c, k} L_{w, c, k}^{-1}}{P_{w, c, k}^{i}+\beta_{s} P_{t} h_{w, c, k} \psi_{w, c, k} L_{w, c, k}^{-1}+N_{0}}
$$

\section{Jain's Fairness Index}

The Jain's fairness index $\left(I_{\text {Jain }}\right)$ is a parameter which is used to measure the fairness of user allocation schemes. It is defined as [17]:

$$
I_{\text {Jain }}=N_{F} \sum_{k=1}^{N_{F}} n_{b u}^{2}(k) /\left(\sum_{k=1}^{N_{F}} n_{b u}(k)\right)^{2}
$$

where $0<I_{\text {Jain }} \leq 1$ and $0 \leq n_{b u}(k) \leq N_{b}$ denotes the number of RBs that the SBS $f_{k}$ allocates to its users. Higher values of $I_{\text {Jain }}$ indicate more efficient load balancing among SBSs.

\section{Proposed User Association ANd Power AllocAtion}

\section{A. Problem Formulation}

Let $\mathbf{X}$ denote $N_{u} \times N_{b} \times N_{F}$ dimensional binary variable which represents the status of RBs in SBS. $\mathbf{X}(m, c, k)=1$ indicates that the user $u_{m}$ is served by the SBS $f_{k}$ in the RB $b_{c}$. Also, we consider the matrix $\mathbf{P}$ with the same size as $\mathbf{X}$, where $\mathbf{P}(m, c, k)$ is the transmit power of the SBS $f_{k}$ in the RB $b_{c}$ to serve the user $u_{m}$. Our aim is to obtain the optimum values of $\mathbf{P}$ and $\mathbf{X}$ that maximize Jain's fairness index and spectral efficiency $\left(\eta_{S E}\right)$ as follows:

$$
\begin{aligned}
& {\left[\mathbf{P}^{*}, \mathbf{X}^{*}\right]=\underset{\mathbf{P}, \mathbf{X}}{\arg \max }\left(I_{\text {Jain }}\right)} \\
& {\left[\mathbf{P}^{*}, \mathbf{X}^{*}\right]=\underset{\mathbf{P}, \mathbf{X}}{\arg \max }\left(\eta_{S E}\right)}
\end{aligned}
$$

subject to the following constraints:

$$
\begin{array}{ll}
\mathbf{S}_{1} \cdot & \sum_{m=1}^{N_{u}} \mathbf{X}(m, c, k) \leq 2, \forall(c, k) \\
\mathbf{S}_{2} \cdot & \sum_{k=1}^{N_{F}} \sum_{c=1}^{N_{b}} \mathbf{X}(m, c, k) \leq 1, \forall m \\
\mathbf{S}_{3} . & \mathbf{P}(m, c, k) \leq P_{\max }, \forall(m, c, k) \\
\mathbf{S}_{4} \cdot & \mathbf{X}(m, c, k) \leq\{0,1\}, \forall(m, c, k) \\
\mathbf{S}_{5} \cdot & \gamma_{m}>\gamma_{m}^{t h}, \forall m
\end{array}
$$

$$
\Omega_{m}=\left\{\left(f_{k}, b_{n}\right) \mid P_{m, n, k}^{O} \geq P_{t h}^{r s r p} \& x_{n, k}=0, k=1, \ldots, N_{F}, n=1, \ldots, N_{b}\right\}
$$

The constraint $\mathbf{S}_{1}$ demonstrates that the maximum number of users that can be served in each RB of SBS, is two (one user in OMA mode and two users in NOMA mode). Each user can only connect to one SBS and occupy one RB in the target SBS according to the constraint $\mathbf{S}_{2}$. $\mathbf{S}_{\mathbf{3}}$ indicates that the maximum transmit power is $P_{\max }$ and the constraint $\mathbf{S}_{4}$ means that $\mathbf{X}$ is a binary variable. Finally, the constraint $\mathbf{S}_{5}$ states that the received SINR of each user must be higher than the predefined threshold value.

\section{B. Proposed Solution}

Obtaining the optimal solution of the problem presented in (10)-(12) requires huge computational complexity and is timeconsuming. Hence, we propose a MANC-based user association and power allocation scheme in order to maximize load balancing and spectral efficiency.

The proposed solution decomposes the problem presented in (10)-(12) into two sub-problems. The first sub-problem finds the number of idle RBs that each SBS can allocate to the connection requests, to maximize the fairness index and the second sub-problem forms the NOMA groups and allocate RBs to maximize the spectral efficiency.

\section{B.1 Obtaining the number of RBS}

Suppose that the number of occupied RBs in the SBS $f_{k}$ is denoted by $n_{b u}(k)$. The sub-problem for finding the number of RBs that each SBS can allocate to ongoing requests, can be obtained by maximizing Jain's fairness index, that is,

$$
n_{b a}^{*}=\underset{n_{b a}}{\arg \max }\left(I_{\text {Jain }}\right)
$$

subject to:

$$
n_{b a}(k) \leq N_{b}-n_{b u}(k)
$$

where $n_{b a}(k)$ denotes the number of RBs that SBS $f_{k}$ can allocate. The above constraint demonstrates that the maximum number of RBs that SBS $f_{k}$ can allocate to ongoing requests, is equal to the number of idle RBs.

\section{B.2 User association and power control}

The proposed MANC scheme, first constructs the neighbor list for each user. The serving SBS should satisfy the minimum required RSRP for each user. Therefore, to reduce the computational complexity and to guarantee the signal quality at the target SBS, we propose to specify the neighbor list for each user considering the RSRP in OMA mode, since the RSRP in OMA mode is higher than the NOMA mode. The neighbor list of the user $u_{m}$ contains the set of pairs SBSs and RBs that the RSRP of $u_{m}$ from them is higher than the predefined threshold value $P_{t h}^{r s r p}$, which is constructed as follows:

See 
Next, the user $u_{m}$ sorts the pairs of $\Omega_{m}$ based on the RSRP value to obtain the list $\bar{\Omega}_{m}$. After that, the user $u_{m}$ sends connection request to the SBS of the first pair in set $\bar{\Omega}_{m}$.

Each SBS $f_{k}$ collects the connection requests from the users and then constructs the neighbor list $\Lambda_{n, k}$ as:

$$
\Lambda_{n, k}=\left\{u_{m} \mid \bar{\Omega}_{m}(1)==\left(f_{k}, b_{n}\right), m=1, \ldots, N_{u}\right\}
$$

where $\bar{\Omega}_{m}(1)$ denotes the first pair in the set $\bar{\Omega}_{m}$. Therefore, $\Lambda_{n, k}$ contains the set of users that send connection request in the RB $b_{n}$ to the SBS $f_{k}$. This SBS should form the NOMA group or select the OMA-user from them to maximize the spectral efficiency.

It was shown that the spectral efficiency of NOMA transmission is higher than the OMA [18]. Also, it was demonstrated that when the user with the highest channel gain is grouped with the user with the lowest channel gain, spectral efficiency is maximized [18]. Hence, we sort the users belonging to $\Lambda_{n, k}$ according to their channel gains, i.e., $\varphi_{m, n, k}=h_{m, n, k} \psi_{m, n, k} L_{m, n, k}^{-1}$, in descending order to obtain the list $\bar{\Lambda}_{n, k}$. Then, the first and last users of $\bar{\Lambda}_{n, k}$ are considered as the strong and weak users, respectively, to form the NOMA group in the RB $b_{n}$ of the SBS $f_{k}$.

Let $u_{s}$ and $u_{w}$ denote the strong and weak users of $\bar{\Lambda}_{n, k}$, respectively. The spectral efficiency of NOMA group depends on the transmit power gains of users and we should obtain the optimum values for $\beta_{s}$ and $\beta_{w}$. Since $\beta_{w}=1-\beta_{s}$, we only formulate the optimization problem for $\beta_{s}$ as follows

$$
\beta_{s}^{*}=\underset{\beta_{s}}{\arg \max }\left(\log _{2}\left(1+\gamma_{s, c, k}^{N, s}\right)+\log _{2}\left(1+\gamma_{w, c, k}^{N, w}\right)\right)
$$

subject to:

$$
\begin{array}{ll}
\mathbf{S}_{\beta, 1} \cdot & \gamma_{s, c, k}^{N, s} \geq \gamma_{s}^{t h} \\
\mathbf{S}_{\beta, 2} \cdot & \gamma_{w, c, k}^{N, w \rightarrow s} \geq \gamma_{w}^{t h} \\
\mathbf{S}_{\beta, 3} \cdot & \gamma_{w, c, k}^{N, w} \geq \gamma_{w}^{t h}
\end{array}
$$

The constraints $\mathbf{S}_{\beta, 1}, \mathbf{S}_{\beta, 2}$, and $\mathbf{S}_{\beta, 3}$ respectively indicate that the SINRs of the strong user, the weak user at the strong user, weak user should be higher than the predefined threshold values.

We use Lagrange dual function to solve the above optimization problem as

$$
\begin{aligned}
L= & \log _{2}\left(1+\gamma_{s, c, k}^{N, s}\right)+\log _{2}\left(1+\gamma_{w, c, k}^{N, w}\right)+\lambda_{\beta}\left(\beta_{s}+\beta_{w}-1\right) \\
& +\lambda_{s}\left(\gamma_{s, c, k}^{N, s}-\gamma_{s}^{t h}\right)+\lambda_{w \rightarrow s}\left(\gamma_{w, c, k}^{N, w \rightarrow s}-\gamma_{w}^{t h}\right)+\lambda_{w}\left(\gamma_{w, c, k}^{N, w}-\gamma_{w}^{t h}\right)
\end{aligned}
$$

where $\lambda_{\beta}, \lambda_{s}, \lambda_{w \rightarrow s}$, and $\lambda_{w}$ are Lagrange multipliers of constraints on the transmit power gains, spectral efficiencies of strong user, weak user at the strong user and weak user, respectively. The above problem is a convex optimization problem and can be solved by Lagrange multipliers. Therefore, our aim will be to maximize (19). To this end, we differentiate (19) with respect to Lagrange multipliers which results in:

$$
\begin{gathered}
\text { See (20) } \\
\frac{\partial L}{\partial \lambda_{\beta}}=\beta_{s}+\beta_{w}-1 \\
\frac{\partial L}{\partial \lambda_{s}}=\frac{\beta_{s} P_{t} h_{s, c, k} \psi_{s, c, k} L_{s, c, k}^{-1}}{P_{s, c, k}^{i}+N_{0}}-\gamma_{s}^{t h} \\
\frac{\partial L}{\partial \lambda_{w \rightarrow s}}=\frac{\beta_{w} P_{t} h_{s, c, k} \psi_{s, c, k} L_{s, c, k}^{-1}}{P_{s, c, k}^{i}+\beta_{s} P_{t} h_{s, c, k} \psi_{s, c, k} L_{s, c, k}^{-1}+N_{0}}-\gamma_{w}^{t h} \\
\frac{\partial L}{\partial \lambda_{w}}=\frac{\beta_{w} P_{t} h_{w, c, k} \psi_{w, c, k} L_{w, c, k}^{-1}}{P_{w, c, k}^{i}+\beta_{s} P_{t} h_{w, c, k} \psi_{w, c, k} L_{w, c, k}^{-1}+N_{0}}-\gamma_{w}^{t h}
\end{gathered}
$$

Then, by setting (20)-(24) to zero, the optimum value of transmit power gain for the strong user is obtained as follows:

$$
\begin{aligned}
\frac{\partial L}{\partial \beta_{s}}= & \frac{P_{t} h_{s, c, k} \psi_{s, c, k} L_{s, c, k}^{-1}}{\ln (2)\left(P_{s, c, k}^{i}+N_{0}+\beta_{s} P_{t} h_{s, c, k} \psi_{s, k, c} L_{s, c, k}^{-1}\right)}- \\
& +\frac{\beta_{w}\left(P_{t} h_{w, c, k} \psi_{w, c, k} L_{w, c, k}^{-1}\right)^{2}}{\ln (2)\left(\left(P_{w, c, k}^{i}+\beta_{s} P_{t} h_{w, c, k} \psi_{w, c, k} L_{w, c, k}^{-1}+N_{0}\right)^{2}+\beta_{w} P_{t} h_{w, c, k} \psi_{w, c, k} L_{w, c, k}^{-1}\right)} \\
& -\lambda_{\beta}+\lambda_{s} \frac{P_{t} h_{s, c, k} \psi_{s, c, k} L_{s, c, k}^{-1}}{P_{s, c, k}^{i}+N_{0}}-\lambda_{w \rightarrow s} \frac{\beta_{w}\left(P_{t} h_{s, c, k} \psi_{s, c, k} L_{s, c, k}^{-1}\right)^{2}}{\left(P_{s, k, c}^{i}+\beta_{s} P_{t} h_{s, k, c} \psi_{s, k, c} L_{s, k, c}^{-1}+N_{0}\right)^{2}} \\
& +\frac{\beta_{w}\left(P_{t} h_{w, c, k} \psi_{w, c, k} L_{w, c, k}^{-1}\right)^{2}}{\left(P_{w, c, k}^{i}+\beta_{s} P_{t} h_{w, c, k} \psi_{w, c, k} L_{w, c, k}^{-1}+N_{0}\right)^{2}}
\end{aligned}
$$




$$
\beta_{s}=\min \left(\frac{\gamma_{s}^{t h}\left(P_{s, c, k}^{i}+N_{0}\right)}{P_{t} h_{s, c, k} \psi_{s, c, k} L_{s, c, k}^{-1}}, 0.5\right)
$$

and $\beta_{w}=1-\beta_{s}$. After finding the optimum values of $\beta_{s}$ and $\beta_{w}$, satisfactions of the constraints $\mathbf{S}_{\beta, 1}, \mathbf{S}_{\beta, 2}$, and $\mathbf{S}_{\beta, 3}$ are checked. If all subjects are satisfied, the NOMA group $\left\{u_{s}, u_{w}\right\}$ is constructed, otherwise, if $\gamma_{s, n, k}^{O} \geq \gamma_{s}^{\text {th }}$, only $u_{s}$ is served via OMA transmission in the RB $b_{n}$ of the SBS $f_{k}$. This process is repeated for all idle RBs of the SBS $f_{k}$ and their spectral efficiencies are computed. Finally, $n_{b a}(k)$ RBs with the highest spectral efficiency are chosen and the users that send connection request, are served. The remaining users (no served users) should send the connection request to the second SBS in their neighbor list. This process is repeated until all users are covered or there is no RB to allocate.

\section{Computational Complexity}

The computational complexity of the proposed method consists of the complexities of obtaining neighbor list, power allocation, and calculation of Jain's fairness index. To obtain the neighbor list for each user, $N_{F} N_{b}$ comparisons are needed, therefore, obtaining the neighbor list of all users required $N_{F} N_{b} N_{u}$ comparisons. Each FBS requires $n_{b a}(k) N_{u}$ comparisons to develop its neighbor list, hence, $N_{u} \sum_{k=1}^{N_{F}} n_{b a}(k)$ comparisons are required to develop the neighbor list of all FBSs. For the FBS $f_{k}$, power allocation is repeated for each idle RB, hence, $2 \sum_{k=1}^{N_{F}} n_{b a}(k)$ additions, $5 \sum_{k=1}^{N_{F}} n_{b a}(k) \quad$ multiplications, and $\quad \sum_{k=1}^{N_{F}} n_{b a}(k)$ comparisons are needed. Finally, calculating of Jain's fairness index needs $3 N_{F}-3$ additions and $N_{F}+2$ multiplications.

\section{PERformance EVAluation}

Here, the performance of the proposed user association and $\mathrm{RB}$ allocation in the introduced system is assessed. We obtain Jain's fairness index, outage probability, and spectral efficiency for different densities of users and SBSs. The results are provided by averaging $10^{4}$ times Monte Carlo simulations considering the parameters listed in Table 1. Also, the path loss between the SBS and user is computed as

$$
L(\mathrm{~dB})=38.46+20 \log _{10}(d)+0.7 d_{i}+L_{w}
$$

where $d$ is the distance between the SBS and user, $d_{i}$ denotes the nearest available distance from FBS to user which is set to $0.5 \mathrm{~m}$, and $L_{w}$ is the wall loss.

Fig. 2 depicts the Jain's fairness index of the proposed method for different densities of users and SBSs. It is observed that for constant density of users, increasing the density of SBS improves the fairness index. Increasing the number of SBSs provides more resources for ongoing connection request, therefore, prevents the congested which enhances Jain's fairness index. On the other side, increasing the density of users at the constant density of SBSs improves the fairness index.
More number of users occupy more resources in different SBSs and fairness index increases.

TABLE 1. SyStem PARAMETERS USED IN Simulations

\begin{tabular}{ll}
\hline \hline Parameter & Value \\
\hline SBS radius & $15 \sim 30 \mathrm{~m}$ \\
SBS maximum transmit power & $23 \mathrm{dBm}$ \\
Noise power & $-174 \mathrm{dBm} / \mathrm{Hz}$ \\
RB bandwidth & $180 \mathrm{kHz}$ \\
& $\sigma_{s}=8 \mathrm{~dB}$ \\
Standard deviation of shadowing of & $\sigma_{s s}=10 \mathrm{~dB}$ \\
different links & $1.6 \mathrm{bits} / \mathrm{sec} / \mathrm{Hz}$ \\
Minimum SE of strong user & $1.2 \mathrm{bits} / \mathrm{sec} / \mathrm{Hz}$ \\
Minimum SE of weak user & $-72 \mathrm{dBm}$ \\
Threshold value for RSRP & 15 \\
Number of RBs per SBS & 50 \\
Number of available RBs &
\end{tabular}

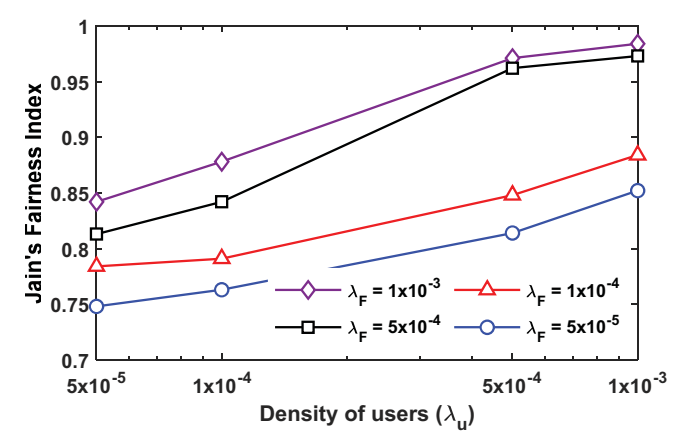

Fig. 2. Jain's fairness index for different densities of users and SBSs.

The effect of the density of users and SBSs on the outage probability is shown in Fig. 3. As the number of users increases at the constant density of SBSs, the greater number of users share the specific RB. As a result, co-channel interference increases and SINR reduces resulting in higher outage probability. While, increasing the number of SBSs at constant number of users enhances the SINR by reducing the density of users in the RBs and outage probability decreases.

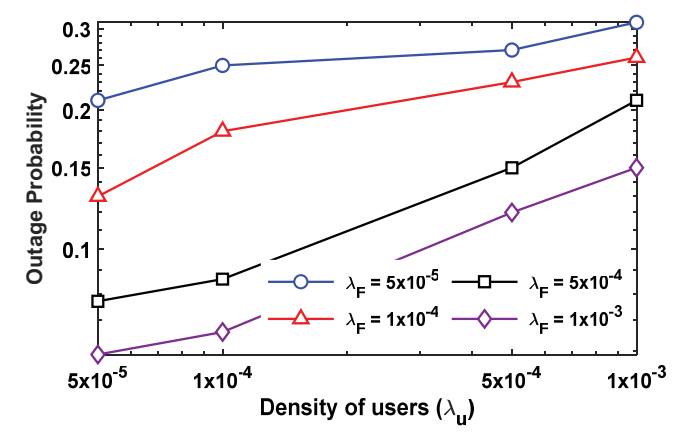

Fig. 3. The effect of the density of users and SBSs on the outage probability. 
According to Fig. 4, increasing the density of SBS at constant density of users increases the spectral efficiency by reducing the SINR. On the other hand, although increasing the density of users at constant density of SBSs reduces the SINR, spectral efficiency increases by the increasing the number of served users.

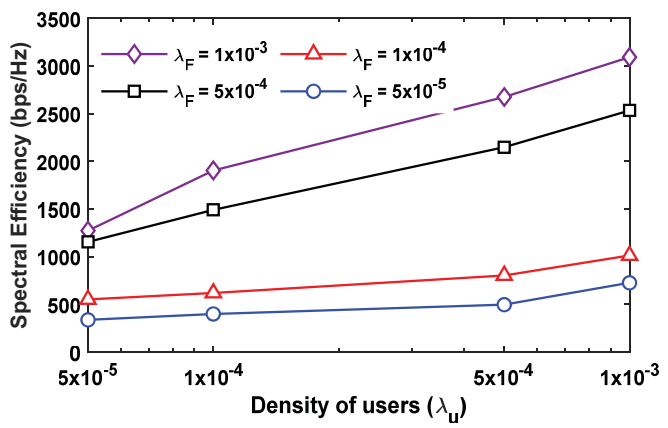

Fig. 4. Spectral efficiency of the proposed user association and RB allocation.

Fig. 5 compares the performance of the proposed method with the conventional method in which each user connects to the SBS that provides the strongest RSRP via OMA mode without considering load balancing. It is observed that the proposed method outperforms the conventional method for different densities of users and SBSs.

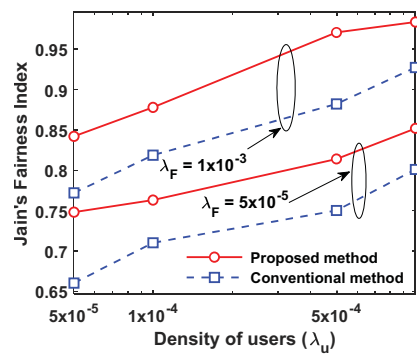

(a)

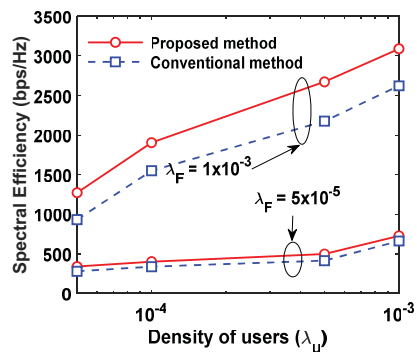

(b)
Fig. 5. Performance comparison between the proposed method and conventional method. (a) Fairness index and (b) spectral efficiency.

\section{CONCLUSION}

In this paper, we proposed a scheme for MANC-based user association and $\mathrm{RB}$ allocation for maximization of load balancing and spectral efficiency in the $5 \mathrm{G}$ of cellular HetNets. The proposed problem was decomposed into sub-problems. To reduce the computational complexity at the SBS, each user identifies its neighbor SBS and then sends connection request to the best SBS. The first sub-problem determines the number of RBs that SBS can allocate to the connection requests such that Jain's fairness index is maximized. Each SBS forms the NOMA group or selects the best OMA user, depending on the channel condition, in each idle RB. Then, the top-ranked RBs based on the spectral efficiency are chosen to maximize the fairness index. Simulation results show that increasing the density of the users and SBSs increases the fairness index and sum rate, while reduces the outage probability. Further, the proposed method outperforms the conventional scheme.

\section{REFERENCES}

[1] J. Zhao, Y. Liu, K. K. Chai, A. Nallanathan, Y. Chen, and Z. Han, "Spectrum allocation and power control for non-orthogonal multiple access in HetNets," IEEE Transactions on Wireless Communications, vol. 16, no. 9, pp. 5825-5837, 2017.

[2] Z. Ding, X. Lei, G. K. Karagiannidis, R. Schober, J. Yuan, and V. K. Bhargava, "A survey on non-orthogonal multiple access for $5 \mathrm{G}$ networks: Research challenges and future trends," IEEE Journal on Selected Areas in Communications, vol. 35, no. 10, pp. 2181-2195, 2017.

[3] D. Liu et al., "User association in 5G networks: A survey and an outlook," IEEE Communications Surveys \& Tutorials, vol. 18, no. 2, pp. 1018-1044, 2016.

[4] P. Swami, V. Bhatia, S. Vuppala, and T. Ratnarajah, "On user offloading in NOMA-HetNet using repulsive point process," IEEE Systems Journal, vol. 13, no. 2, pp. 1409-1420, 2018.

[5] H.-S. Jo, Y. J. Sang, P. Xia, and J. G. Andrews, "Outage probability for heterogeneous cellular networks with biased cell association," in 2011 IEEE Global Telecommunications Conference-GLOBECOM 2011, 2011, pp. 1-5: IEEE.

[6] E. Hossain, M. Rasti, H. Tabassum, and A. Abdelnasser, "Evolution towards 5G multi-tier cellular wireless networks: An interference management perspective," arXiv preprint arXiv:1401.5530, 2014.

[7] A. Alizadeh and M. Vu, "Load balancing user association in millimeter wave MIMO networks," IEEE Transactions on Wireless Communications, 2019.

[8] I. Guvenc, "Capacity and fairness analysis of heterogeneous networks with range expansion and interference coordination," IEEE Communications Letters, vol. 15, no. 10, pp. 1084-1087, 2011.

[9] H. Zhou, S. Mao, and P. Agrawal, "Approximation algorithms for cell association and scheduling in femtocell networks," IEEE Transactions on Emerging Topics in Computing, vol. 3, no. 3, pp. 432-443, 2015.

[10] D. Fooladivanda, A. Al Daoud, and C. Rosenberg, "Joint channel allocation and user association for heterogeneous wireless cellular networks," in 2011 IEEE 22nd International Symposium on Personal, Indoor and Mobile Radio Communications, 2011, pp. 384-390: IEEE.

[11] K. Shen and W. Yu, "Distributed pricing-based user association for downlink heterogeneous cellular networks," IEEE Journal on Selected Areas in Communications, vol. 32, no. 6, pp. 1100-1113, 2014.

[12] R. Madan, J. Borran, A. Sampath, N. Bhushan, A. Khandekar, and T. Ji, "Cell association and interference coordination in heterogeneous LTE-A cellular networks," IEEE Journal on selected areas in communications, vol. 28 , no. 9, pp. 1479-1489, 2010.

[13] R. Sun, M. Hong, and Z.-Q. Luo, "Joint downlink base station association and power control for max-min fairness: Computation and complexity," IEEE Journal on Selected Areas in Communications, vol. 33, no. 6, pp. 1040-1054, 2015.

[14] V. N. Ha and L. B. Le, "Distributed base station association and power control for heterogeneous cellular networks," IEEE Transactions on Vehicular Technology, vol. 63, no. 1, pp. 282-296, 2013.

[15] M. Hong and Z.-Q. Luo, "Distributed linear precoder optimization and base station selection for an uplink heterogeneous network," IEEE Transactions on Signal Processing, vol. 61, no. 12, pp. 3214-3228, 2013.

[16] M. Feng, T. Jiang, D. Chen, and S. Mao, "Cooperative small cell networks: High capacity for hotspots with interference mitigation," IEEE Wireless Communications, vol. 21, no. 6, pp. 108-116, 2014.

[17] A. B. Sediq, R. H. Gohary, R. Schoenen, and H. Yanikomeroglu, "Optimal tradeoff between sum-rate efficiency and Jain's fairness index in resource allocation," IEEE Transactions on Wireless Communications, vol. 12, no. 7, pp. 3496-3509, 2013.

[18] Z. Ding, P. Fan, and H. V. Poor, "Impact of user pairing on 5G nonorthogonal multiple-access downlink transmissions," IEEE Transactions on Vehicular Technology, vol. 65, no. 8, pp. 6010-6023, 2015. 